\title{
Outcomes of Routine perioperative Nursing Care for Women Undergoing Gynecologic Cancer surgeries
}

\author{
Aya M. Abdelhafez ${ }^{1}$, Nabila Taha ${ }^{2} \&$ Amal Fouad Aref Ahmed ${ }^{3}$ Hisham Ahmed El-Sayed Abou-Taleb ${ }^{4}$ \\ 1. Assistant lecturer, Department of Maternal \& Newborn Health Nursing, Faculty of Nursing, Assiut University, \\ Egypt. \\ 2. Professors of Gynecology and Obstetrics Nursing, Faculty of Nursing, Assiut University, Egypt. \\ 3. Assistant Professors of Gynecology and Obstetrics Nursing, Faculty of Nursing, Assiut University, Egypt. \\ 4. Assistant Professors of obstetrics \& gynecology, Faculty of medicine, Assiut University, Egypt.
}

\begin{abstract}
Background: surgery for gynecological cancer still represents a main line of therapy in almost all cases. Perioperative management can significantly decrease patient morbidity and mortality. This study aimed to: assess the outcomes of routine perioperative nursing care for women undergoing gynecologic cancer surgeries. Patients and methods: an observational research design was used in this study. A sample of 25 women was recruited randomly; who have undergone gynecological cancer surgeries at Woman's Health Hospital, Assiut University from the start of January 2019 to the end of June 2019. Results: the postoperative complications represent $68.0 \%$ of women; the most common complications were the overall surgical site infection which represented about $64.0 \%$. Conclusions: The routine perioperative nursing care for gynecologic surgery is not sufficient to improve patient outcomes and prevent postoperative complications. Recommendations: applying high-level evidence-based, preoperative assessment and intervention is required to reduce patient's morbidity after gynecologic cancer surgeries.
\end{abstract}

\section{Keywords: Outcomes, Gynecologic Cancer \& Perioperative Routine Nursing Care.}

\section{Introduction}

Gynecological cancers refer to cancers of the cervix, ovaries, endometrium, vulva, and vagina. Every year, there are over one million new cases worldwide caused by the three major gynecological cancers (cervical, endometrial, and ovarian cancer) (Rhoda Suubi Muliira et al., 2016).

Perioperative period refers to around the time of surgery. More specifically, the period of time extending from the time the patient goes into the hospital, clinic, or doctor's office for surgery until the time the patient goes home. Perioperative period includes the three phases of surgery: preoperative, intraoperative, and postoperative (www.cancer.gov). Perioperative care reflects a significant component of national health-care utilization and expenditures with an estimated 8.4 million discharges associated with a surgical procedure, and a cost estimate of greater than 157-billion dollars. The management and treatment of gynecologic cancer patients' is complex and should recognize patient outcomes (PROs) (Meyer et al., 2018; Klafke et al., 2019)

Among gynecologic cancer patients; Major extensive surgery still represents a corn-stone of therapy in almost all cases annually.

There is no any therapeutic intervention without consequences; therefore, it's imperative to know the possible complications related to the perioperative period before undertaking surgery (Committee on Gynecologic Practice, 2018).

Complications' rates after gynecologic surgeries are affected by patients' age, obesity, physical \& medical conditions, and the provided perioperative care. Failure to identify those factors may negatively affect surgical outcomes, length of hospital stay and increased morbidity and mortality (Arora \& Somashekhar, 2018, Nelson et al., 2019).

Surgical site infection (SSI) is an established quality indicator and predictor for adverse patient outcomes. SSIs are the most common infections found among hospitalized patients in all gynecologic surgery. SSIs lead to increased hospital stay, hospitalization costs, increased readmission to hospital after discharge, reoperations and overall morbidity and mortality (Anthony et al., 2011, Bakkum-Gamez et al., 2013, Mahdi et al., 2014, Tanner et al., 2015, Johnson et al., 2016; Taylor et al., 2017, Agarwal et al., 2019, Gezer et al., 2020, Martinez et al., 2020).

The nurse's role in improving postoperative patient's outcomes is comprehensive. The nurse plays a crucial part in promoting the implementation of evidence-based practices, providing counseling and education during the perioperative period (Green, 2015). 


\section{Significance of the study}

The burden of gynecological cancer in developing countries appears huge account for $25 \%$ of all new cancers diagnosed among women aged up to 65 years compared to $16 \%$ in the developed countries. According to a recent report; developing countries accounted for 820,265 cases $(77.7 \%)$ of global estimates for new cases of the commonest gynecological cancer including cervical, corpus and ovarian cancer in 2009. In 2014; in Egypt about 426 cases diagnosed with uterine cancer, 2434 case with ovarian cancer, 752 cases with cervical cancer, 56 cases with vulvar carcinoma, and 103 cases with vaginal cancer (Fatma Nady et al., 2018).

Nurses caring for patients in the pre, intra and, post-operative period have an important role in advising individuals about the risks associated with surgical outcomes including SSI and how it should be managed. Knowledge and practices of nurses play a key role for the prevention of surgical complications. To achieve good surgical outcomes, it is very important for nurses to have good knowledge and practice towards prevention of surgical problems (Haleema et al., 2017).

Surgery is the cornerstone of the treatment of gynecologic malignancies (GM). High complexity surgeries for gynecologic cancer are associated with the substantial risk of SSIs, high perioperative morbidity, and poor overall survival (Agarwal et al., 2019).

SSIs were reported as the most common hospitalacquired infections (HAIs) by the American College of Surgeons (ACS). Up to $60 \%$ of these infections are estimated to be preventable by proper preoperative nursing care. SSI accounts for approximately $20 \%$ of documented HAIs and is the most costly (Agarwal et al., 2019, Mengesha et al., 2020)

To date, novel preoperative processes have been investigated and implemented, but despite having good face validity, clear effects of these processes on important clinical outcomes remain limited (Cui et al., 2017). Therefore the current study was done to investigate the outcomes of routine perioperative nursing care for women undergoing gynecologic cancer surgeries.

\section{Aim of the study:}

This study aimed to assess the outcomes of routine perioperative nursing care for women undergoing gynecologic cancer surgeries.

\section{Patients \& Methods}

\section{Technical design}

\section{Research design}

An observational research design was used in this study.

\section{Research question}

What are the outcomes of routine perioperative nursing care for women undergoing gynecologic cancer surgeries?

\section{Setting of the study}

This study was conducted at the inpatient gynecological department, major operations \& postoperative units at Woman's Health Hospital, Assiut University.

\section{Sample size:}

According to the follow rate of the gynecological cancer patients at Woman's Health Hospital, Assiut University and by the using sample size equitation; a sample of 25 women was recruited randomly; who have undergone gynecological cancer surgeries when they met the inclusion criteria during the study period. The sample size calculated by:

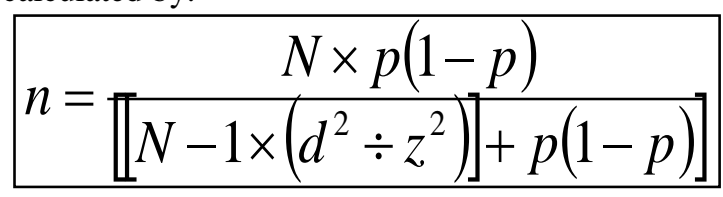

$\mathrm{N}=$ total patient population size of 50 who attended the gynecology department at Assiut university hospitals during year 2019 - 2020. $\mathrm{Z}=$ confidence levels is 0.95 and is equal to $1.96 \mathrm{D}=$ the error ratio is $=0.05, \mathrm{P}=$ the property availability ratio and neutral $=0.50$

\section{Study outcomes}

- Primary outcomes: the routine nursing care done \& the occurrence of SSIs (superficial, deep and organ SSI).

- Secondary outcomes: length of hospital stay, hospital readmission, \& other postoperative complications.

\section{Inclusion criteria}

- All women undergoing gynecologic cancer surgeries who agreed participation in the study.

- Age from 18 up to 65 years.

\section{Tools of the study}

In this study; data were collected through perioperative assessment checklist which developed by the investigator after reviewing the related literatures. This checklist consisted of four parts:

Part 1: women's demographic characteristics; age, level of education, occupation, BMI......etc.

\section{Part 2: preoperative checklist:}

- Women's Medical comorbidities; such as cardiovascular diseases, respiratory diseases, diabetes, chronic renal or hepatic disease, immune deficiency diseases.

\section{- Preoperative nursing preparations perceived}

Part 3: intraoperative checklist:

- Intraoperative data: surgical indication, length of surgery (LOS: defined as a time from surgical incision to closure), assessment of blood loss, intraoperative blood transfusion, total fluid given during the operation, and requiring bowel 
resection......etc.

- Intraoperative nursing preparations perceived

Part 4: postoperative and discharge data:

- Postoperative outcomes and discharge data: postoperative temperature, postoperative complications; the development of SSI, length of hospital stays, and readmission within the postdischarge period.

- Postoperative nursing preparations perceived

\section{Operational design}

Preparatory phase

The researcher reviewed related literature (local \& international) using textbooks, web articles and scientific magazines then the tools were prepared.

\section{Content validity}

The content validity was used to assess the study tools. Item-level Content Validity Index (I-CVI) was calculated by a panel of five experts rating each scale's item for its relevance to the construct of health care. The ratings were on a 4-point scale with a response format of $1=$ not relevant to $4=$ highly relevant. The I-CVI for each item was computed based on the percentage of experts giving a rating of 3 or 4 , indicating item relevance. The content validity index for the total scale (S$\mathrm{CVI}$ ), calculated by averaging the I-CVI responses from the five experts and dividing by the number of items, was equal to .96. A rating of .90 is considered to be an acceptable standard for an $\mathrm{S}$ CVI.

\begin{tabular}{|c|c|c|c|c|}
\hline $\begin{array}{c}\text { No of items } \\
\text { of the } \\
\text { study tool }\end{array}$ & $\begin{array}{c}\text { Scoring system } \\
\text { of items by } \\
\text { panel experts }\end{array}$ & $\begin{array}{c}\text { Total } \\
\text { I-CVI }\end{array}$ & $\begin{array}{c}\text { S- } \\
\text { CVI/ } \\
\text { AVE }\end{array}$ & $\begin{array}{c}\text { S- } \\
\text { CVI } \\
\text { /UA }\end{array}$ \\
\hline 33 item & $\begin{array}{c}29 \text { item take } \\
\text { score 1 }\end{array}$ & 32 & 0.96 & 0.9 \\
\cline { 2 - 2 } & $\begin{array}{c}4 \text { items score } \\
\text { ranged from } \\
0.6-0.8\end{array}$ & & & \\
& \multicolumn{2}{|c}{} & & \\
\hline
\end{tabular}

\section{Reliability}

The internal consistency of the tool scale was calculated by using Cronbach's Alpha; and it was 0.841 .

\section{Pilot study}

A pilot study was carried out after validation of the study tool on $10 \%$ of women who were undergone gynecologic cancer surgeries to test the clarity and feasibility of tool. After conducting the pilot study there weren't any modification on the tool and so the sample of the pilot study was included in the total sample.

Field work

The study was carried out from the start of January 2019 to the end of June 2019.

\section{Ethical considerations}

- Oral informed consent was obtained from women who accepted participation in the study, after explaining the nature and purpose of the study.

- There was no any risk for the women during conduction of the study.

- The study was followed common ethical principles in clinical research.

- Confidentiality and anonymity would be assured.

- The participating women had the right to refuse participation or withdraw from the study without any rational at any stage of the study.

Administrative design

An approval was obtained from the ethical committee and the dean of the Faculty of Nursing, Assiut University. An official permission also was obtained from the vice manager of the Woman's Health Hospital, Assiut University to proceed with this study \& collect data.

\section{Procedures}

- Women were interviewed a day before the operation to explain the nature \& purpose of the study and gave them full description and information needed about the study; then oral informed consent was obtained.

- Women who accepted participation in the study were observed for pre, intra and postoperative nursing care provided to them by using checklist. The outcomes (routine nursing care done and the occurrence of SSIs, length of hospital stay, hospital readmission, and post-operative complications were observed and recorded.

\section{Preoperative checklist}

- Started in the night a day before $\&$ the morning of the operation by using preoperative checklist.

- The investigator was recording all of the demographic information.

- Assessment of medical comorbidities as; cardiovascular \& pulmonary diseases, diabetes, chronic renal or hepatic disease......etc.

- Recording the information of complete abdominal \& pelvic examinations which done by the physician.

- The weight and height of women were measured by the investigator to calculate the BMI.

- Recording if the operation informed consent was filled by the women or not.

- Then recording the preoperative preparations which done by the nurse as:

- NPO (nothing per mouth); allow fluids up to 2 hours, light breakfast 6 hours \& heavy meal 8 hours prior to surgery, measuring vital signs, giving preoperative medications, performing enema, inserting a Foley's catheter, monitoring of blood glucose \& providing clean hospital gowns to the 
women before the woman going to the operating room.

\section{Intraoperative check list}

The investigator recording the following by using checklist:

Helping of the nurse in positioning of women in the operating table, sterilize women's skin with betadine antiseptic solution, assessment of vital signs, monitoring blood oxygenation levels, giving fluid therapy, antibiotics, necessary medications \& blood transfusion if required, monitoring the amount of blood loss and recording the length of operation.

\section{Postoperative check list}

The investigator recording the following by using checklist:

- Initial \& ongoing nursing assessment of patients' airway patency, effectiveness of respiration and circulatory status, vital signs, wound condition, performing dressings, checking and removing drains when ordered.

- Nursing assessment of the fluid balance; including IV fluids, output from catheters \& drains and the ability to void, assessment of level of consciousness and pain after surgery, giving postoperative medications and follow up through visiting the hospital about 2 weeks after discharge.

- The postoperative follow up was done immediately after the operation then within 48 or 72 hours after the operation and finally; within 30 days post-operation to measure the delayed postoperative complications.

\section{Statistical analysis}

Data were collected, coded and analyzed by using SPSS 20 statistical software. Uni-variable analysis was used through presentation of continuous variables as mean and standard deviation when normally distributed and median and range when abnormally distributed. Categorical variables (nominal \& ordinal) were presented as frequency and percentage.

\section{Results}

Table (1): Distribution of women according to their demographic data.

\begin{tabular}{|c|c|c|}
\hline \multirow{2}{*}{ Variables } & \multicolumn{2}{|c|}{ Total } \\
\hline & No. $(n=25)$ & $\%$ \\
\hline \multicolumn{3}{|l|}{ Age (years) } \\
\hline Range & \multicolumn{2}{|c|}{$18-69$} \\
\hline Mean \pm SD & \multicolumn{2}{|c|}{$50.92 \pm 12.958$} \\
\hline Median & \multicolumn{2}{|c|}{53} \\
\hline \multicolumn{3}{|l|}{ Residence } \\
\hline Rural & 25 & 100.0 \\
\hline Urban & 0 & 0.0 \\
\hline \multicolumn{3}{|l|}{ Occupation } \\
\hline Employed & 1 & 4.0 \\
\hline House wife & 24 & 96.0 \\
\hline \multicolumn{3}{|l|}{ Educational level } \\
\hline Illiterate & 20 & 80.0 \\
\hline Read and write & 2 & 8.0 \\
\hline Secondary & 2 & 8.0 \\
\hline University & 1 & 4.0 \\
\hline \multicolumn{3}{|l|}{ Marital Status } \\
\hline Single & 2 & 8.0 \\
\hline Married & 17 & 68.0 \\
\hline Divorced & 1 & 4.0 \\
\hline Widow & 5 & 20.0 \\
\hline \multicolumn{3}{|l|}{ Parity } \\
\hline Nulliparous & 5 & 20.0 \\
\hline Multipara & 12 & 48.0 \\
\hline Grandmultipara & 8 & 32.0 \\
\hline
\end{tabular}

(SD: standard deviation) 
Table (2): Distribution of women according to their preoperative data

\begin{tabular}{|c|c|c|}
\hline \multirow{2}{*}{ Variables } & \multicolumn{2}{|c|}{ Total } \\
\hline & No. $(n=25)$ & $\%$ \\
\hline \multicolumn{3}{|l|}{ Weight (kg) } \\
\hline Range & \multicolumn{2}{|c|}{$42-115$} \\
\hline Mean \pm SD & \multicolumn{2}{|c|}{$70.680 \pm 14.7443$} \\
\hline \multicolumn{3}{|l|}{ BMI } \\
\hline Range & \multicolumn{2}{|c|}{$18.67-39.79$} \\
\hline Mean \pm SD & \multicolumn{2}{|c|}{$27.3933 \pm 4.59982$} \\
\hline Normal weight & 6 & 24.0 \\
\hline Overweight & 19 & 76.0 \\
\hline \multicolumn{3}{|l|}{ Medical Diseases } \\
\hline \multicolumn{3}{|l|}{ Cardiac events } \\
\hline None & 23 & 92.0 \\
\hline Coronary artery disease & 1 & 4.0 \\
\hline Idiopathic heart disease & 1 & 4.0 \\
\hline \multicolumn{3}{|l|}{ Cardiovascular risk factors } \\
\hline None & 12 & 48.0 \\
\hline Hypertension & 13 & 52.0 \\
\hline \multicolumn{3}{|l|}{ Respiratory diseases } \\
\hline None & 22 & 88.0 \\
\hline Asthma & 1 & 4.0 \\
\hline Pulmonary fibrosis \& bilateral pleural effusion & 1 & 4.0 \\
\hline COPD & 1 & 4.0 \\
\hline \multicolumn{3}{|l|}{ Hepatic diseases } \\
\hline No & 24 & 96.0 \\
\hline $\mathrm{HCV}$ & 1 & 4.0 \\
\hline \multicolumn{3}{|l|}{ Diabetes mellitus } \\
\hline No & 16 & 64.0 \\
\hline Controlled DM & 9 & 36.0 \\
\hline \multicolumn{3}{|l|}{ Previous operations } \\
\hline No & 15 & 60.0 \\
\hline Yes & 10 & 40.0 \\
\hline \multicolumn{3}{|l|}{ Preoperative chemotherapy } \\
\hline No & 15 & 60.0 \\
\hline Yes & 10 & 40.0 \\
\hline
\end{tabular}

Note: (SD: standard deviation, BMI: body mass index, COPD: chronic obstructive pulmonary disease, HCV: hepatitis $C$ virus, DM: diabetes mellitus).

Table (3): Checklist of the preoperative nursing preparations perceived

\begin{tabular}{|c|c|c|}
\hline \multirow{2}{*}{ Variables } & \multicolumn{2}{|c|}{ Total } \\
\hline & Done & Not done \\
\hline Assessment of medical comorbidities & Done & \\
\hline Performing complete abdominal \& pelvic examinations & Done & \\
\hline Assessment of preoperative laboratory values & Done & \\
\hline Measuring the weight and height of women & Done & \\
\hline $\begin{array}{l}\text { Performing physical preparations including: Nutrition and fluids: NPO } \\
\text { (nothing per mouth); allow fluids up to } 2 \text { hours, light breakfast } 6 \text { hours \& } \\
\text { heavy meal } 8 \text { hours prior to surgery. }\end{array}$ & Done & \\
\hline Measuring vital signs & Done & \\
\hline Giving preoperative medications & Done & \\
\hline Performing enema and inserting a Foley's catheter & Done & \\
\hline Monitoring, controlling of blood glucose \& providing clean hospital gowns & Done & \\
\hline Providing preoperative patient education & & Not done \\
\hline $\begin{array}{l}\text { Encouraging the women to shower with antibacterial soap and antiseptic } \\
\text { solution the night and the morning before operation }\end{array}$ & & Not done \\
\hline Providing sterile cloths to the women at morning of the operation & & Not done \\
\hline Hair removal & & Not done \\
\hline
\end{tabular}


Table (4): Distribution of women according to their intraoperative data

\begin{tabular}{|c|c|c|}
\hline \multirow{2}{*}{ Variables } & \multicolumn{2}{|c|}{ Total } \\
\hline & No. $(n=25)$ & $\%$ \\
\hline \multicolumn{3}{|l|}{ Surgical diagnosis } \\
\hline Vulval cancer & 2 & 8.0 \\
\hline Vaginal cancer & 1 & 4.0 \\
\hline Endometrial cancer & 8 & 32.0 \\
\hline Ovarian cancer & 10 & 40.0 \\
\hline Cervical cancer & 3 & 12.0 \\
\hline Uterine sarcoma & 1 & 4.0 \\
\hline \multicolumn{3}{|l|}{ Disease stage } \\
\hline 0 & 1 & 4.0 \\
\hline IA1 & 4 & 16.0 \\
\hline IA2 & 2 & 8.0 \\
\hline IB & 3 & 12.0 \\
\hline $\mathrm{IC}$ & 4 & 16.0 \\
\hline IIA & 1 & 4.0 \\
\hline IIB & 1 & 4.0 \\
\hline IIIA & 2 & 8.0 \\
\hline IIIB & 3 & 12.0 \\
\hline IIIC & 1 & 4.0 \\
\hline IV & 3 & 12.0 \\
\hline \multicolumn{3}{|l|}{ Surgical approach } \\
\hline Laparotomy & 24 & 96.0 \\
\hline Laparoscopy & 1 & 4.0 \\
\hline \multicolumn{3}{|c|}{$\begin{array}{l}\text { Length of surgery from incision } \\
\text { to closure (hrs.) }\end{array}$} \\
\hline Range & \multicolumn{2}{|c|}{$1-9$} \\
\hline Mean \pm SD & \multicolumn{2}{|c|}{$4.04 \pm \quad 2.071$} \\
\hline \multicolumn{3}{|c|}{ Estimated blood loss during operation } \\
\hline Class $\mathrm{I}<750$ & 12 & 48.0 \\
\hline Class II $=750-1500$ & 5 & 20.0 \\
\hline Class III $=1500-2000$ & 5 & 20.0 \\
\hline Class IV=>2000 & 3 & 12.0 \\
\hline \multicolumn{3}{|c|}{ Intraoperative blood transfusion } \\
\hline No & 8 & 32.0 \\
\hline Yes & 17 & 68.0 \\
\hline \multicolumn{3}{|c|}{ Volume of fluids given during operation } \\
\hline $1000-2000$ & 11 & 44.0 \\
\hline $2500-4000$ & 13 & 52.0 \\
\hline $4500-6000$ & 1 & 4.0 \\
\hline \multicolumn{3}{|l|}{ Presence of surgical drains } \\
\hline No & 5 & 20.0 \\
\hline yes & 20 & 80.0 \\
\hline
\end{tabular}

Table (5): Checklist of intraoperative nursing preparations perceived.

\begin{tabular}{|l|l|l|}
\hline \multicolumn{1}{|c|}{ Variables } & \multicolumn{2}{c|}{ Total } \\
\cline { 2 - 3 } & Done & Not done \\
\hline The nurse helps the women in positioning in the operating room. & Done & \\
\hline Sterilize women's skin with betadine antiseptic solution. & Done & \\
\hline $\begin{array}{l}\text { Assessment of women's vital signs, giving anesthesia, maintaining blood } \\
\text { oxygenation levels, giving fluid therapy, antibiotics, necessary medications \& } \\
\text { blood transfusion if required. }\end{array}$ & Done & \\
\hline Complete coverage of incisional area with sterile dressing & Done & \\
\hline Sterile closing tray for fascia and skin closure & & Not done \\
\hline Staff glove change (and gown if soiled) before fascia closure & & Not done \\
\hline Maintain normothermia in the theater & Done & \\
\hline
\end{tabular}


Table (6): Distribution of women according to their postoperative outcomes and discharge data

\begin{tabular}{|c|c|c|}
\hline \multirow{2}{*}{ Variables } & \multicolumn{2}{|c|}{ Total } \\
\hline & No. $(n=25)$ & $\%$ \\
\hline \multicolumn{3}{|l|}{ Postoperative temperature } \\
\hline Normal & 23 & 92.0 \\
\hline Hyperthermia & 2 & 8.0 \\
\hline \multicolumn{3}{|c|}{ Postoperative blood transfusion } \\
\hline No & 15 & 60.0 \\
\hline Yes & 10 & 40.0 \\
\hline \multicolumn{3}{|l|}{ Postoperative complications } \\
\hline No & 8 & 32.0 \\
\hline Yes & 17 & 68.0 \\
\hline \multicolumn{3}{|c|}{$\begin{array}{l}\text { Classification of complications } \\
\text { Accordion grade classifications }\end{array}$} \\
\hline Non & 8 & 32.0 \\
\hline Mild & 9 & 36.0 \\
\hline Moderate & 5 & 20.0 \\
\hline Sever & 1 & 4.0 \\
\hline Death & 2 & 8.0 \\
\hline \multicolumn{3}{|c|}{ Length of hospital stay (days) } \\
\hline Range & \multicolumn{2}{|c|}{$2-30$} \\
\hline Mean \pm SD & \multicolumn{2}{|c|}{$8.48 \pm 7.171$} \\
\hline Median & & 7 \\
\hline \multicolumn{3}{|c|}{ Readmission within the post-discharge period } \\
\hline No & 21 & 84.0 \\
\hline Yes & 4 & 16.0 \\
\hline
\end{tabular}

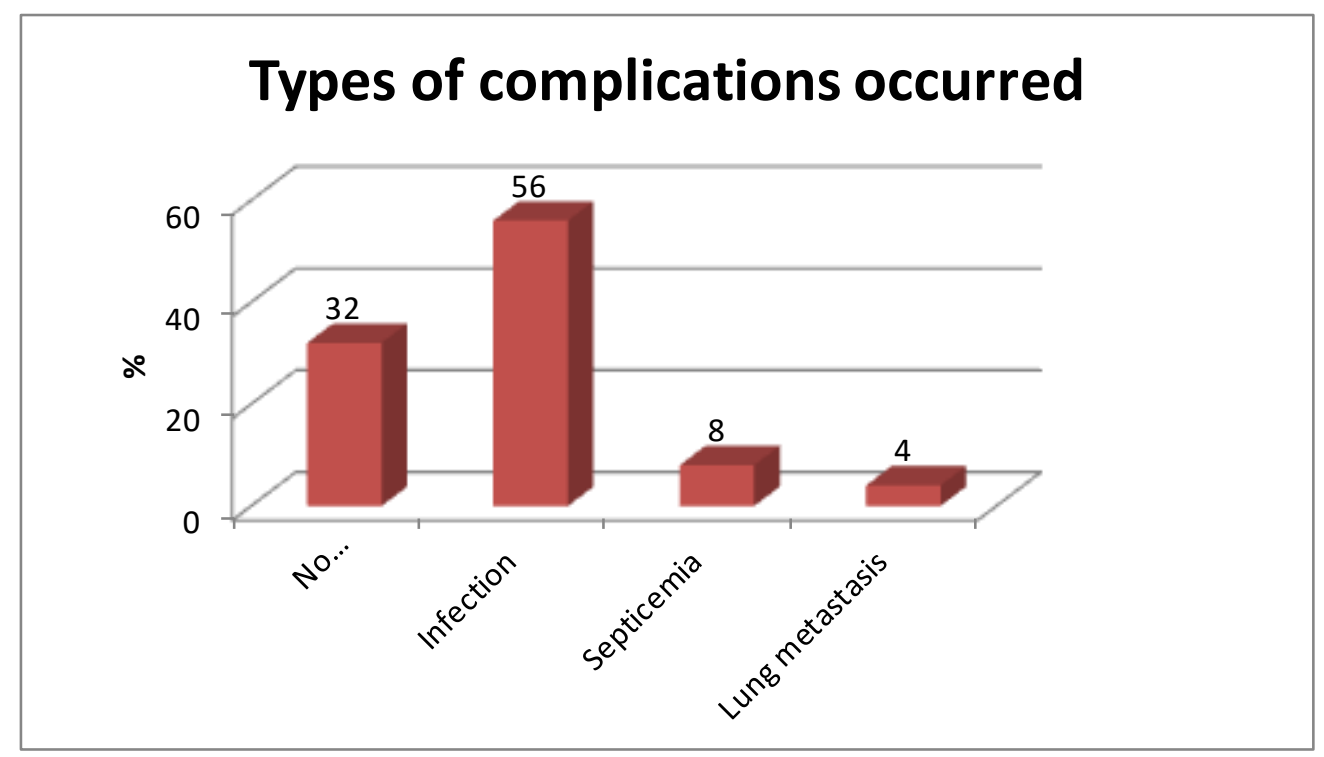

Figure (1): Distribution of women according to of post- operative complications occurred 
Table (7): Checklist of postoperative nursing care

\begin{tabular}{|l|c|c|}
\hline \multicolumn{1}{|c|}{ Variables } & \multicolumn{2}{c|}{ Total } \\
\cline { 2 - 3 } & \multicolumn{1}{|c|}{ Done } & Not done \\
\hline $\begin{array}{l}\text { Initial \& ongoing nursing assessment of airway patency, effectiveness of } \\
\text { respiration and circulatory status. }\end{array}$ & Done & \\
\hline Taken vital signs & Done & \\
\hline $\begin{array}{l}\text { Assessing wound condition; dressings; and checking and removing drains } \\
\text { when ordered. }\end{array}$ & Done & \\
\hline $\begin{array}{l}\text { Assessed the fluid balance; including IV fluids, output from catheters and } \\
\text { drains and the ability to void. }\end{array}$ & Done & \\
\hline Assessment of level of consciousness and pain after surgery. & Done & \\
\hline Giving postoperative medications. & Done & \\
\hline $\begin{array}{l}\text { The women were followed up through visiting the hospital about 2 weeks } \\
\text { after discharge. }\end{array}$ & Done & \\
\hline Good hand hygiene before and after dealing with patient & & Not done \\
\hline Hand-cleansing agent readily available & & Not done \\
\hline Dressing removal within 24-48 hours & & Not done \\
\hline Patient education on wound care and infection symptoms & Not done \\
\hline Dismiss patient with antibacterial soap and antiseptic solution & Not done \\
\hline Follow-up phone call within 24-72 hours & & done \\
\hline
\end{tabular}

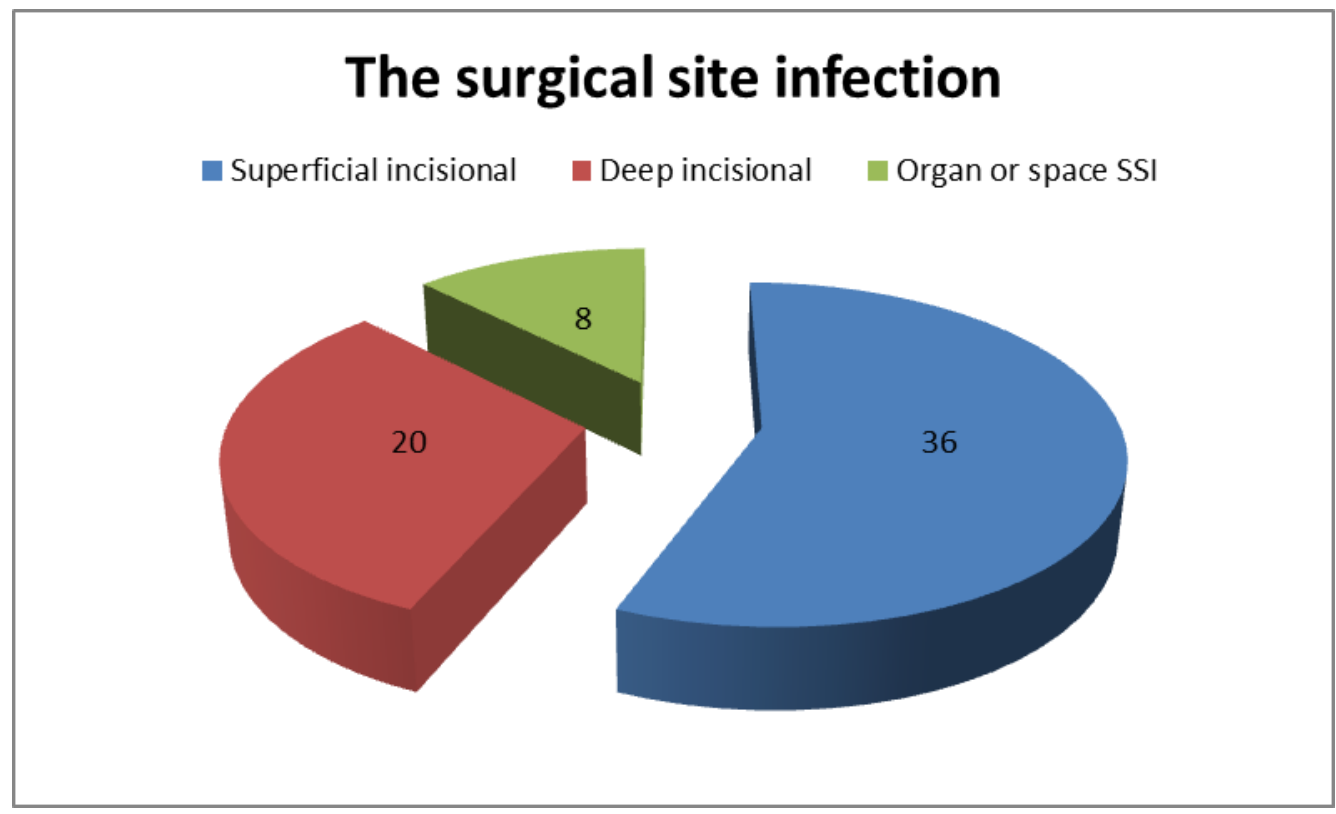

Figure (2): Distribution of women according to SSI development.

Table (1): Distribution of women according to their demographic data; this table show that the Mean \pm SD age of the study women was $50.92 \pm 12.958$, all women $(100.0 \%)$ were live in rural areas, $96.0 \%$ of them were house wives and $80.0 \%$ were illiterate.

Table (2): Distribution of women according to their preoperative data; it shows that about $76 \%$ of women were overweight and $36.0 \%$ of them were diabetic.

Table (3): Illustrates the preoperative nursing preparations perceived; in which there were defect in the preoperative care provided as encouraging the women to take preoperative (night \& morning) shower.
Table (4): Distribution of women according to their intraoperative data; this table illustrates that the $96.0 \%$ of women had laparotomy surgical intervention procedure. Ovarian cancer represented the most common type of gynecologic cancer $40.0 \%$ and length of surgery from incision to closure was ranged from 1-9 hours. The table also illustrates that $48.0 \%$ of estimated blood loss during operation was class $\mathrm{I}<750.68 .0 \%$ of women were received intraoperative blood transfusion. Table (5): Shows checklist of intraoperative nursing preparations perceived; in which there were defect in the intraoperative care provided as using a sterile closing tray for fascia and skin closure. 
Table (6): Distribution of women according to their postoperative outcomes and discharge data; it shows that $68.0 \%$ of women had postoperative complications; ranged from hyperthermia to stroke \& lung metastasis. It also shows that the length of hospital stays ranged from 2-30 days with the Mean \pm SD was $8.48 \pm 7.171$ days and also the hospital readmission within the post-discharge period was $16.0 \%$.

Fig. (1): Shows the distribution of women as regard the type of postoperative complications; it was observed that most common complications was infection which represented $56.0 \%$ and septicemia was $8 \%$ of all complications occurred.

Table (7): Shows the postoperative nursing care perceived; there were defect in the postoperative care as good hand hygiene before and after dealing with patient.

Fig. (2): Distribution of women according to SSI development in which the superficial incisional infection constituted the most common type (36\%).

\section{Discussion}

Preoperative assessment and education have been shown to improve postoperative outcomes for patients with gynecologic cancers. Increases in psychological distress and surgical complications in gynecologic cancer women have been attributed to inadequate preoperative assessment, preparation, and planning, as well as limited access for staff members (Fang Huang et al., 2018). The current study has assessed the outcomes of perioperative nursing care provided for the women undergoing gynecologic cancer surgeries.

According to postoperative complications, the present study found that the most postoperative complications were surgical site infections that constituted about two thirds of women. The results of the present study came in alignment with Cham et al., (2019); who were studied the factors related to short-term, significant perioperative morbidity and mortality for ovarian cancer women undergoing surgery and to make a nomogram to predict the danger of adverse perioperative outcomes. Their study administrated on 7029 women; they found that the most common complications that occurred were sepsis. But the current study disagreed with Bicer et al., (2019); who carried out a study to assess the risk of surgery for gynecological oncologic patients and suggest an easy risk assessment model and risk reduction by applying findings. They found that of the 258 patients, two-thirds of them had no complications and therefore the commonest complication was the acid-base imbalance less than one fifth, followed by urinary tract infection nearly one tenth. Also, the current study was not agreed with Szender et al., (2015); who evaluate the performance of the National Surgical Quality Improvement Program (NSQIP) Universal Surgical Risk Calculator
(URC) on the patients of a gynecologic oncology service. This study administered within the USA; they reviewed 628 consecutive surgeries performed by gynecologic oncology service between July 2012 and June 2013. Their results concluded that SSI was not accurately predicted. These differences may came from the differences in the parameters associated with complications as performance status, ascites, operation length, type of surgery, patient characteristics, operating room, and the surrounding characteristics.....etc. also the difference may come from the difference in the sample size. The dissimilarities between the current and the previous studies came from different in circumstances between countries as patient characteristics differ according to region. Also, the current study agreed with (Gouda \& Sayed, 2018); study who conducted a study on problems encountered among patients undergoing hysterectomy and nursing implications in the department of gynecology, at Zagazig University hospital over a one-year period from June 2015 to the end of May 2016. Their study revealed that the routine group had more postoperative complications than the study group. The similarities come from that guidelines help to improve perioperative care for patients undergoing gynecologic/oncology operations.

The current study revealed that the routine perioperative routine nursing care is not sufficient to decrease postoperative complications and this agreed with (Deeb, 2019); whose study conducted to assess the effect of perioperative protocol of care on clinical outcome among patients undergoing coronary artery bypass graft at National Heart Institute in Cairo and in open heart surgical intensive care unit in Shebin El Kom Teaching Hospital at Menoufia governorate. Deep study concluded that there was a significant improvement clinical outcome among study group who receive the protocol of care than control group who didn't receive it. So the current study and Deep study agreed with each other in the point that despite the type of surgery the routine perioperative care is not sufficient and it should be enhanced by introducing of evidence based care programs.

Regarding the length of hospital stay, the current study found that the median length of hospital stays was one weak which comes nearly close to the results of Shah et al., (2017); who evaluated the effect of enhanced recovery on length of hospital stay, readmissions and, identified risk factors for readmission. Their study recruited 707 women who underwent colorectal procedures between 2011 and 2015 in Virginia. 383 pre enhanced recovery patients were compared to 324 patients following protocol implementation. The median length of hospital stay in their study was longer in the pre than the post-enhanced recovery implementation. Also, the present study agreed with Cham et al., 
(2019); they found that women who stay $>3$ days represented more than three- quarters. The current study also agreed with Huang et al., (2015); who use a descriptive design to describe an advanced practice $\mathrm{RN}$-led preoperative assessment and education clinic designed to improve the quality of preoperative preparation and postoperative outcomes of patients with gynecologic cancer. They used a convenience sample of women with confirmed or suspected gynecologic cancer aged 15 years or older who visited the clinic from December 2014 to February 2015 prior to their surgery. Huang et al. concluded that the median length of hospital stays for patients undergoing surgeries was nearly one week before the implementation of the program. These similarities between the current and the previous studies were indicates that the routine perioperative nursing care is not sufficient and must be supported by special programs to decrease postoperative complications. Also, the current study agreed with (Gouda \& Sayed, 2018); Their study revealed that the length of hospital stay is increased in control group than study group. These similarities between both studies came to the insufficiency of the routine perioperative care in reduction of the length of hospital stay. Also the circumstances of both studies were nearly close to each other.

Concerning the 30 days readmission to the hospital, the present study found that about less than one fifth of women readmitted to the hospital after discharge. This finding nearly agreed with Shah et al., (2017); they found that the Thirty-day readmission was nearly one fifth in the preenhanced recovery pathway. This finding also agreed with Martinez et al., (2020); their study aimed to assess the impact of established protocol on SSI after colon surgery in 2017 performing a retrospective analysis of 2 years (2016-2017) and then data were collected prospectively before and after the conduction of the protocol. They found that the pre-intervention group tends to have higher readmission rate than the post- intervention group. The similarities between the studies came from the insufficiency of the routine perioperative care in reduction of hospital readmission after discharge from the hospital.

As regards the length of surgery, the current study found that the mean length of surgery was four hours. This comes close to the study carried by Aletti et al., (2007); who evaluated the impact of patients' age and American Society of Anesthesiologists (ASA) and, surgical complexity score (SCS) on short-term morbidity and overall survival. Whose results revealed that the mean length of surgery was 3.55 hours.

Concerning the type of surgery performed, the present study agreed with Szender et al., (2015); Both; the current study and Szender et al. study found that the most common surgeries performed were a total abdominal hysterectomy with bilateral salpingo-oophorectomy (radical hysterectomy). The rational of this similarity is the possibility of the radical hysterectomy to prevent or decrease the metastasis of the cancer. Also, this study agreed with (Gouda \& Sayed, 2018); Ahmed's study revealed that the most common surgical approach done for their patients was abdominal hysterectomy. The agreement between both studies is come from popularity of the abdominal hysterectomy in Egypt than vaginal and laparoscopic approach.

Concerning the body mass index (BMI), the present study found that more than three quarters of the women were obese. This result was not in alignment with Wu et al., (2016); who investigated the influence of gynecologic oncologists on treatment outcomes for cervical cancer patients treated by radical hysterectomy between January 2005 and June 2010 in China. A total of 839 patients were included. Their results regarding the BMI found that less than half of women in both groups were overweight. These results may be slightly differed from the current study due to cultural differences as obesity is very common in the Arabic population than in other populations. The finding of the current study was agreed with Cham et al., (2019); their study found that obesity was more common among all recruited women.

\section{Conclusion}

\section{The current study concluded that}

- Surgical site infection (SSI) is a most common postoperative complication.

- The routine perioperative nursing care is not sufficient to produce satisfied patient outcomes and prevent postoperative complications for women undergoing gynecologic cancer surgeries.

\section{Recommendations}

The present study recommended the following:

- Applying high-level evidence-based perioperative nursing care for gynecologic cancer surgeries as bundled care.

- Train nurses on the implementation of highlevel evidence-based nursing interventions.

- Conducting future more researches regarding this topic on large number of patients to generalize the results.

\section{Strength of the study}

The main strength of this study was the recommendation of a simple, practical, and convenient tool for perioperative nursing care in patients with gynecologic cancer. Other strength is the random allocation of women to the study.

\section{Limitations of the study}

The main limitation of the study was that results cannot be generalized for the whole population of 
patients with gynecologic cancer. Refused participation in the study from some women was another limitation.

\section{Acknowledgment}

Big thanks to all of the women for their participation in this study; praying to God for them and all patients with a speedy recovery. The investigator extends special thanks to nurses and staff members of Obstetrics and Gynecology in Women's Health Hospital, Assiut University, for their assistance have offered in the data collection during the study period.

\section{References}

- Agarwal R., Sannappavar, Appukuttan A., Ashok A., \& Rajanbabu A., (2019): A prospective study evaluating the impact of implementing 'bundled interventions' in reducing surgical site infections among patients undergoing surgery for gynaecological malignancies. Eur J Obstet Gynecol Reprod Biol, 243, 21-25.

- Aletti G., Dowdy S., Podratz K., \& Cliby W., (2007): Relationship among surgical complexity, short-term morbidity, and overall survival in primary surgery for advanced ovarian cancer. Am J Obstet Gynecol, 197(6), 676 e1-7.

- Anthony T., Murray B., Sum-Ping J., Lenkovsky F., Vornik V., \& Parker B., (2011): Evaluating an evidence-based bundle for preventing surgical site infection: A randomized trial. Archives of Surgery, 146(3), 263-9.

- Bakkum-Gamez J., Dowdy S., Borah B., Haas L., Mariani A., \& Martin J., (2013): Predictors and costs of surgical site infections in patients with endometrial cancer. Gynecol Oncol, 130(1), 100-6.

- Bicer C., Raoufi J., Iscan S., Guney M., \& Erdemoglu E., (2019): Surgical risk assessment for gynecological oncologic patients. Turk J Obstet Gynecol, 16(3), 15863.

- Cham S., Chen L., St Clair C., Hou J., Tergas A., \& Melamed A., (2019): Development and validation of a riskcalculator for adverse perioperative outcomes for women with ovarian cancer. Am J Obstet Gynecol, 220(6), 571 e1-71 e8.

- Cui H., Turney B., \& Griffiths J., (2017): The preoperative assessment and optimization of patients undergoing major urological surgery. Curr Urol Rep, 18(7), 54.

- Deeb S., \& Hussein, (2019): Effect of perioperative protocol of care on clinical outcome among patients undergoing coronary artery bypass graft. In Shehata \& Fareed (Eds.).
- Fang Huang, Yen Y., Chia, Chui L., Eng, Yong K., Lim, Kwai L., Yam, Soh C., |\& Tan, (2018): Evaluation of a preoperative clinic for women with gynecologic cancer. Clinical Journal of Oncology Nursing, 19(6), 769-72.

- Fatma Nady, Mamdouh El-Sherbiny, Entisar Youness, \& Hassan Hanan Elzeblawy, (2018): Effectiveness of quality of life planned teaching program on women undergoing gynecologic cancer treatment. American Research Journal of Oncology, 1(1), 1-17.

- Gezer S., Yalvac H., Gungor K., \& Yucesoy I., (2020): Povidone-iodine vs chlorhexidine alcohol for skin preparation in malignant and premalignant gynaecologic diseases: A randomized controlled study. European Journal of Obstetrics, Gynecology, and Reproductive Biology, 244, 45-50.

- Gouda E., Ahmed, \& Sayed R., (2018). Problems encountered among patients undergoing hyesterecetomy and nursing implications. The Egyptian Journal of Health Care, 9(4).

- Green Linda, (2015): Preventing surgicalsite infections. AmericanNurseToday, 10(9).

- Haleema, Robina K., Muhammad A., Ali W., \& Syed A., Gilani. (2017): Assessment of nurses' knowledge and practices regarding prevention of surgical site infection. Saudi Journal of Medical and Pharmaceutical Sciences, 3(6B), 585-95.

- Huang Fang, Chia Yen, Eng Chui, Lim Yong, Yam Kwai, Tan Soh, (2015): Evaluation of a preoperative clinic for women with gynecologic cancer. Clinical Journal of Oncology Nursing, 769-72.

- Johnson M., Kim S., Langstraat C., Jain S., Habermann E., \& Wentink J., (2016): Using bundled interventions to reduce surgical site infection after major gynecologic cancer surgery. Obstet Gynecol, 127(6), 1135-44.

- Klafke N., Mahler C., von Hagens C., Uhlmann L., Bentner M., \& Schneeweiss A., (2019): The effects of an integrated supportive care intervention on quality of life outcomes in outpatients with breast and gynecologic cancer undergoing chemotherapy: Results from a randomized controlled trial. Cancer Med, 8(8), 3666-76.

- Mahdi H., Gojayev A., Buechel M., Knight J., SanMarco J., Lockhart \& D., (2014): Surgical site infection in women undergoing surgery for gynecologic cancer. International Journal of Gynecological Cancer, 24(4), 779-86. 
- Martinez C., Omesiete P., Pandit V., Thompson E., Nocera M., \& Riall T., (2020). A protocol-driven reduction in surgical site infections after colon surgery. Journal of Surgical Research, 246, 100-05.

- Mengesha A., Tewfik N., Argaw Z., Beletew B., \& Wudu M., (2020): Practice of and associated factors regarding prevention of surgical site infection among nurses working in the surgical units of public hospitals in addis ababa city, ethiopia: A cross-sectional study. PLoS One, 15(4), e0231270.

- Meyer L., Lasala J., Iniesta M., Nick A., Munsell M., \& Shi Q., (2018). Effect of an enhanced recovery after surgery program on opioid use and patient-reported outcomes. Obstet Gynecol, 132(2), 281-90.

- Nelson G., Bakkum-Gamez J., Kalogera E., Glaser G., Altman A., \& Meyer L., (2019): Guidelines for perioperative care in gynecologic/oncology: Enhanced recovery after surgery (eras) society recommendations-2019 update. Int J Gynecol Cancer, 29(4), 651-68.

- Rhoda Suubi Muliira, Jansirani Natarajan, \& Gloria Vergara, (2016): A review of interventions to enhance the quality of life for gynaecological cancer patients. Clinical Obstetrics, Gynecology and Reproductive Medicine, 2(5), 235-43.

- Shah P., Johnston L., Sarosiek B., Harrigan A., Friel C., \& Thiele R., (2017): Reducing readmissions while shortening length of stay: The positive impact of an enhanced recovery protocol in colorectal surgery. Dis Colon Rectum, 60(2), 219-27.

- Szender J., Frederick P., Eng K., Akers S., Lele S., \& Odunsi K., (2015): Evaluation of the national surgical quality improvement program universal surgical risk calculator for a gynecologic oncology service. Int J Gynecol Cancer, 25(3), 51220.

- Tanner J., Padley W., Assadian O., Leaper D., Kiernan M., \& Edmiston C., (2015): Do surgical care bundles reduce the risk of surgical site infections in patients undergoing colorectal surgery? A systematic review and cohort meta-analysis of 8,515 patients. Surgery, 158(1), 66-77.

- Taylor J., Marten C., Munsell M., Sun C., Potts K., \& Burzawa J., (2017): The disinfect initiative: Decreasing the incidence of surgical infections in gynecologic oncology. Annals of Surgical Oncology, 24(2), 362-68.

- Wu M., Li J., Lu H., Wang L., Zhang B., \& Lin Z., (2016): Impact of the care provided by gynecologic oncologists on outcomes of cervical cancer patients treated with radical hysterectomy. Onco Targets Ther, 9, 1361-70. 